\title{
Atomically thin InSe: A high mobility two-dimensional material
}

\author{
FENG Wei ${ }^{1,2}$, ZHENG Wei ${ }^{1,2}$, GAO Feng ${ }^{1,2} \&$ HU PingAn ${ }^{1 *}$ \\ ${ }^{1}$ Key Lab of Microsystem and Microstructure, Harbin Institute of Technology, Harbin 150080, China; \\ ${ }^{2}$ School of Materials Science and Engineering, Harbin Institute of Technology, Harbin 150080, China
}

Received December 17, 2016; accepted January 19, 2017; published online March 3, 2017

Since silicon is limited by its physical properties, it is challenging and important to find candidate materials for high performance electronic devices. Two-dimensional (2D) semiconductor materials have attracted dramatically increasing interest due to their unique physical, electronic and optical properties and great promise for next generation high performance nanoelectronics and optoelectronic devices [1]. 2D semiconductor materials are compatible to silicon based COMS processing, such as photolithography for large-scale fabrication. Atomically ultrathin thickness is good for better electrostatic control of electrical conductivity and significant device downscaling for high density integration. Atomically flat surface without dangling bonds makes 2D semiconductor materials free from carrier scattering caused by surface roughness, leading to $2 \mathrm{D}$ semiconductor materials based electronic devices could outperform silicon devices in scaling limitation. A number of studies have been focused on filed effect transistors (FETs), a core unit building block of electronic logic circuit, using 2D $\mathrm{MoS}_{2}$ and black phosphorus as channel materials [2,3]. However, the further experiment demonstrated that $\mathrm{MoS}_{2}$ is not a good candidate material for high performance electronic devices due to its heavier electron effective mass $\left(m^{*}=0.45 m_{0}\right)$ and relatively low electron mobility [4]. Few-layer black phosphorus show a high mobility of $1000 \mathrm{~cm}^{2} \mathrm{~V}^{-1} \mathrm{~s}^{-1}$ at room temperature [3]. However, it is extremely unstable and sensitive to oxygen and water, which limit its practical applications [5]. So, it is important to explore new 2D semiconductor materials for electronic nanodevices.

Indium selenide ( $\mathrm{InSe}$ ) is a typical layered semiconductor, belonging to III-VI group compounds. The Figure 1(a) is

*Corresponding author (email: Hupa@hit.edu.cn) the crystal structure of InSe. It is constructed of vertically stacked Se-In-In-Se sheets and each sheet is weakly bound to neighboring sheets by van der Waals force. Unlike indirect bandgap of $\mathrm{MoS}_{2}$, InSe is a direct bandgap material. The most attracting properties are its lighter electron mass $\left(m^{*}=0.143\right.$ $m_{0}$ ) [6] and high electron mobility of $10^{3} \mathrm{~cm}^{2} \mathrm{~V}^{-1} \mathrm{~s}^{-1}$. Recently, our and others studies reveal that thin-film InSe is a promising material for high performance electronic and optoelectronic devices [7-10]. The mobility of multilayer InSe FETs is up to $10^{3} \mathrm{~cm}^{2} \mathrm{~V}^{-1} \mathrm{~s}^{-1}$ due to suppressed carrier scattering from dielectric substrate [7]. Furthermore, few-layered InSe photodetector shows a high responsivity [9] and gatetunable behavior [10], making promising application in photodetection. However, the early studies still focus on multilayer or thin-film (10 nm) [7-10], atomically thin InSe is been ignored. Recently, Bandurin et al. [11] reported a high electron mobility and anomalous photoluminescence (PL) phenomenon in atomically thin InSe. The electron mobility is measured to be $10^{3}$ and $10^{4} \mathrm{~cm}^{2} \mathrm{~V}^{-1} \mathrm{~s}^{-1}$ at room temperature and $4 \mathrm{~K}$, respectively (as shown in Figure 1(b)). The devices based on monolayer InSe show good qualities and stabilities under ambient environment.

To circumvent the influence of chemical stability, few-layer InSe was encapsulated using exfoliated BN. Few layer graphene (FEG) servers as contact metal to InSe nanosheets. The electron density $(n)$ in InSe was effectively controlled by top and bottom gate voltages. The contact resistance between InSe and FLG depended on gate voltage due to the changes in the Schottky barrier height (SBH) depending on doping level. The mobility of few layers InSe filed-effect devices (FED) showed almost independent of temperature $(T)$, which was limited by disorder. The best FED showed an ultrahigh electron mobility of $12700 \mathrm{~cm}^{2} \mathrm{~V}^{-1} \mathrm{~s}^{-1}$. A faster 

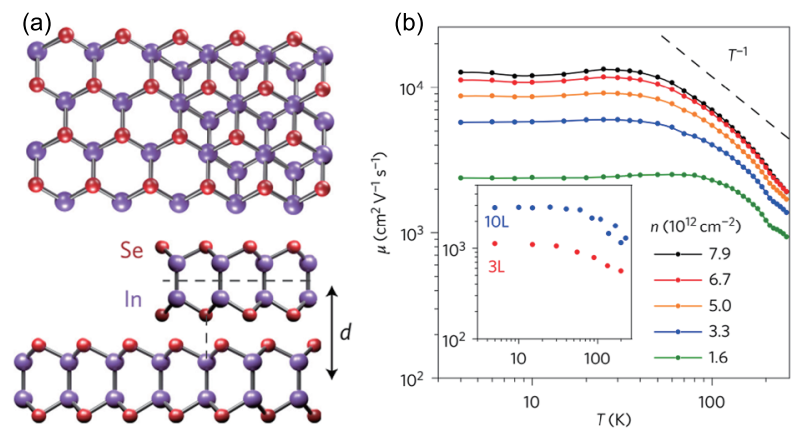

Figure 1 (Color online) (a) Crystal structure of InSe. Purple and red spheres correspond to indium and selenium atoms, respectively; (b) $T$ dependence of the Hall mobility $\mu$ for the $6 L$ device.

decreased mobility than standard $T^{-1}$ was observed for $T>$ $100 \mathrm{~K}$, attributing to additional scattering at homopolar optical phonons. The room temperature mobility of few layer InSe FED decreased to $1000 \mathrm{~cm}^{2} \mathrm{~V}^{-1} \mathrm{~s}^{-1}$, which was comparable to other 2D semiconductors, such as $\mathrm{MoS}_{2}$ and black phosphorus. Further, the quantum Hall effect was explored in few layers InSe system. For the six-layer InSe device, Shubnikov de Haas oscillations ( $\mathrm{SdHO}$ ) gave a quantum mobility of about $2200 \mathrm{~cm}^{2} \mathrm{~V}^{-1} \mathrm{~s}^{-1}$. The $\rho_{x x}$ diminished and $\rho_{x y}$ exhibited plateau in high $B$ due to $\mathrm{SdHO}$ developed into the quantum Hall effect. The values of electron density measured by SdHO were consistent with measured by Hall effect, indicating all conduction electrons occupy a single electric subband and a single valley. The values of effective electron mass were $(0.14 \pm 0.01) m_{0}$ for six-layer InSe and $(0.17 \pm 0.02) m_{0}$ for three-layer InSe. The PL spectrums were studied to gain more information few-layer layers InSe. Unlike few layer InSe showed two peaks, monolayer InSe showed only the higher energy peak. The peak exhibited a blue shift as thickness decreasing. The disappearance of lower energy peak in monolayer InSe was attributed to the monolayer's mirror-plane symmetry.
In summary, for the first time, the atomically thin InSe FED was successfully demonstrated and they showed a high performance in electronic and optoelectronic devices. Taking into account the environment stability and high room temperature mobility, 2D InSe was more promising candidate for further nanoelectronics.

This work was supported by the National Key Basic Research Program of China (Grant No. 2013CB632900), National Natural Science Foundation of China (Grant Nos. 61390502 \& 21373068), the Foundation for Innovative Research Groups of the National Natural Science Foundation of China (Grant No.51521003), and by Self-Planned Task of State Key Laboratory of Robotics and System (HIT) (Grant No. SKLRS201607B).

1 Wang Q H, Kalantar-Zadeh K, Kis A, et al. Electronics and optoelectronics of two-dimensional transition metal dichalcogenides. Nat Nanotech, 2012, 7: 699-712

2 Radisavljevic B, Radenovic A, Brivio J, et al. Single-layer $\mathrm{MoS}_{2}$ transistors. Nat Nanotech, 2011, 6: 147-150

3 Li L, Yu Y, Ye G J, et al. Black phosphorus field-effect transistors. Nat Nanotech, 2014, 9: 372-377

4 Yoon Y, Ganapathi K, Salahuddin S. How good can monolayer $\mathrm{MoS}_{2}$ transistors be? Nano Lett, 2011, 11: 3768-3773

5 Liu H, Neal A T, Zhu Z, et al. Phosphorene: An unexplored 2D semiconductor with a high hole mobility. ACS Nano, 2014, 8: 4033-4041

6 Kuroda N, Nishina Y. Resonance Raman scattering study on exciton and polaron anisotropies in InSe. Solid State Commun, 1980, 34: 481-484

7 Feng W, Zheng W, Cao W, et al. Back gated multilayer InSe transistors with enhanced carrier mobilities via the suppression of carrier scattering from a dielectric interface. Adv Mater, 2014, 26: 6587-6593

8 Sucharitakul S, Goble N J, Kumar U R, et al. Intrinsic electron mobility exceeding $10^{3} \mathrm{~cm}^{2} /(\mathrm{V} \mathrm{s})$ in multilayer InSe FETs. Nano Lett, 2015, 15: 3815-3819

9 Tamalampudi S R, Lu Y Y, Kumar U. R, et al. High performance and bendable few-layered InSe photodetectors with broad spectral response. Nano Lett, 2014, 14: 2800-2806

10 Feng W, Wu J B, Li X, et al. Ultrahigh photo-responsivity and detectivity in multilayer InSe nanosheets phototransistors with broadband response. J Mater Chem C, 2015, 3: 7022-7028

11 Bandurin D A, Tyurnina A V, Yu G L, et al. High electron mobility, quantum Hall effect and anomalous optical response in atomically thin InSe. Nat Nanotech, 2016, doi: 10.1038/nnano.2016.242 